\title{
DYNAMIC OUTPUT FEEDBACK CONTROL OF MINIMUM-PHASE NONLINEAR PROCESSES
}

\author{
PRODROMOS DAOUTIDIS and COSTAS KRAVARIS \\ Department of Chemical Engineering, The University of Michigan, Ann Arbor, MI 48109-2136, U.S.A.
}

(Received for publication 6 August 1991)

\begin{abstract}
This paper concerns the synthesis of dynamic output feedback controllers for minimum-phase nonlinear processes. The problem is addressed first for open-loop stable and then for general minimumphase nonlinear processes, leading to one- and two-degree-of-freedom controllers, respectively. The synthesis of the controllers essentially involves combination of state feedback and state observers. An input/output interpretation of the resulting control structures illustrates the importance of alternative state-space realizations of the process inverse for the controller implementation. Internal stability conditions are derived for the closed-loop system. Simulation studies in a chemical reactor example illustrate the application of the control methodology developed.
\end{abstract}

\section{INTRODUCTION}

Nonlinear control has emerged as a research area of rapidly increasing activity during recent years. This was motivated by the need to deal directly with the nonlinear nature of processes and was facilitated by the increasing computational capabilities and availability of nonlinear process models. Two major approaches can be identified in the relevant literature:

(1) The input/output approach, which deals with nonlinear systems as input/output operators.

(2) The state-space approach, which deals explicitly with the state-space description of nonlinear systems.

The input/output approach in a nonlinear setting is essentially an abstract operator approach. It has mainly been used to study stability and feedback properties, using operator methods and/or functional analysis methods [e.g. Safonov (1980) and Desoer and Vidyasagar (1975)]. From a synthesis point of view, however, the input/output approach cannot lead to analytical controller formulas, given the abstract nature of nonlinear operators. Therefore, numerical control algorithms have been proposed for minimumphase open-loop stable processes (Economou et al., 1986; Parrish and Brosilow, 1988) and special cases of open-loop unstable processes (Cheng and Brosilow, 1987; Hidalgo and Brosilow, 1990). The above approach is in contrast with results in linear systems, where the transfer function description captures essential process information (e.g. poles and zeros) and allows the derivation of explicit dynamic output feedback controllers in the form of transfer functions. In a nonlinear setting, all useful process information is hidden in the state-space description of the process. Consequently, the problem of synthesis of nonlinear dynamic output feedback controllers becomes the problem of deriving state-space realizations of the controllers, and, therefore, state-space considerations must necessarily be taken into account for its solution.
The state-space approach for nonlinear systems is based on the mathematical and conceptual framework of differential geometry. In this framework, key elements of linear control theory have recently found their nonlinear analogs [e.g. nonlinear inversion, Hirschorn (1979) and zero dynamics, Byrnes and Isidori (1985)]. Furthermore, the notion of input/output linearization through state feedback has provided an explicit controller synthesis framework for minimum-phase nonlinear processes (Kravaris and Chung, 1987). The controller synthesis formulas that have been developed, however, are predominantly based on state feedback, i.e. the controller implementation requires measurements of the process state variables. On the other hand, the state feedback results have provided valuable insight on the nature of the output feedback control problem. A combination of state feedback and open-loop state observer has been proposed for open-loop stable minimum-phase processes (Kravaris and Chung, 1987), leading to a dynamic output feedback controller. Despite the above result, however, the output feedback control problem has not been directly and systematically addressed in a general nonlinear setting.

Motivated by the above considerations, the objectives of the present work are:

(1) to solve the dynamic output feedback control problem for general minimum-phase nonlinear processes;

(2) to interpret the resulting controllers from an input/output perspective.

With regard to the first objective, the synthesis of the controllers will be based on combination of state feedback controllers and state observers. This approach seems the most logical, given the power and explicitness of the state-space methods for nonlinear systems. With regard to the second objective, an input/output interpretation of the control structures developed will illustrate the importance of alternative 
state-space realizations of the process inverse operator for the controller implementation, establishing. thus, a precise and rigorous connection between the state-space and input/output approaches. More specifically, in the rest of the paper we will consider single-input/single-output (SISO) minimum-phase nonlinear processes. Following a brief discussion of the input/output and state-space approaches in nonlinear control, we will formulate precisely the dynamic output feedback controller synthesis problem, and we will solve it initially for open-loop stable processes and then for general nonlinear processes. The issue of closed-loop stability will be discussed and precise internal stability conditions will be derived. Finally, simulation studies in a chemical reactor example will illustrate the application of the control methodology developed.

\section{PRELIMINARIES}

The dynamic behavior of chemical processes is typically described by a set of nonlinear ordinary differential equations. These equations, together with the specification of some output variables and some manipulated input variables, constitute the state-space description of the process. The importance of the state-space description in nonlinear processes goes beyond allowing the simulation of the dynamic behavior of the processes: it captures essential information about the process dynamic characteristics (e.g. poles and zeros, in linear systems terminology), and can also be used implicitly or explicitly as part of a control scheme for the process.

In this work, we consider SISO nonlinear processes, with a state-space description of the form:

$$
\begin{aligned}
& \dot{x}=f(x)+g(x) u \\
& y=h(x)
\end{aligned}
$$

where $x \in \mathbb{R}^{n}, u \in \mathbb{R}$ and $y \in \mathbb{R}$ denote the state variable vector, the manipulated input and the output, respectively, $f$ and $g$ denote smooth vector fields on $\mathbb{R}^{n}$, and $h$ denotes a smooth scalar field on $\mathbb{R}^{n}$. For the purpose of the theoretical development, and without loss of generality, we assume that the origin is the equilibrium point of interest. Referring to a nonlinear process of the form of eq. (1), let $r$ be the relative order of $y$ with respect to $u$, i.e. the smallest integer for which $L_{g} L_{f}^{r-1} h(x) \neq 0$. We assume that the relative order $r$ is well defined in an open set $X \subset \mathbb{R}^{n}$ which contains the origin and which will be considered to be the state manifold in the subsequent development. Then, the dynamic system

$$
\begin{aligned}
\dot{w}=f(w)+g(w) & \frac{\frac{\mathrm{d}^{r} y}{\mathrm{~d} t^{r}}-L_{f}^{r} h(w)}{L_{g} L_{f}^{r-1} h(w)} \\
u= & \frac{\mathrm{d}^{r} y}{\mathrm{~d} t^{r}}-L_{f}^{r} h(w) \\
L_{g} L_{f}^{r-1} h(w) &
\end{aligned}
$$

represents a full-order realization of the process inverse (Hirschorn, 1979).

Also, let $t_{1}(x), \ldots, t_{n-r}(x)$ be scalar fields such that

- the scalar fields $t_{1}(x), \ldots, t_{n-r}(x), \quad h(x)$, $L_{f} h(x), \ldots, L_{f}^{r-1} h(x)$ are linearly independent;

- $L_{g} t_{t}(x)=0, i=1, \ldots,(n-r)$.

Then, the nonlinear mapping

$$
\zeta=T(x)=\left[\begin{array}{c}
t_{1}(x) \\
\vdots \\
t_{n-r}(x) \\
h(x) \\
L_{f} h(x) \\
\vdots \\
L_{f}^{r-i} h(x)
\end{array}\right]
$$

qualifies as a curvilinear coordinate system. In this new coordinate system, eq. (1) is transformed into the normal form (Byrnes and Isidori, 1985)

$$
\begin{aligned}
\dot{\zeta}_{1} & =L_{f} t_{1}(\zeta) \\
& \vdots \\
\zeta_{n-r} & =L_{f} t_{n-r}(\zeta) \\
\zeta_{n-r+1} & =\zeta_{n-r+2} \\
& \vdots \\
\zeta_{n-1} & =\zeta_{n} \\
\dot{\zeta}_{n} & =L_{f}^{r} h(\zeta)+L_{o} L_{f}^{r-1} h(\zeta) u \\
y & =\zeta_{n-r+1}
\end{aligned}
$$

where the $\zeta$-dependence in the right-hand side of the above equations implies that the corresponding expressions are evaluated at $x=T^{-1}(\zeta)$. The above normal form allows the calculation of a minimalorder realization of the process inverse, given by the dynamic system

$$
\begin{aligned}
\dot{z}_{1}= & L_{f} t_{1}\left(z_{1}, \ldots, z_{n-r}, y, \frac{\mathrm{d} y}{\mathrm{~d} t}, \ldots, \frac{\mathrm{d}^{r-1} y}{\mathrm{~d} t^{r-1}}\right) \\
\vdots & \\
\dot{z}_{n-r}= & L_{f} t_{n-r}\left(z_{1}, \ldots, z_{n-r}, y, \frac{\mathrm{d} y}{\mathrm{~d} t}, \ldots, \frac{\mathrm{d}^{r-1} y}{\mathrm{~d} t^{r-1}}\right) \\
u= & \frac{\frac{\mathrm{d}^{r} y}{\mathrm{~d} t^{r}}-L_{f}^{r} h\left(z_{1}, \ldots, z_{n-r}, y, \frac{\mathrm{d} y}{\mathrm{~d} t}, \ldots, \frac{\mathrm{d}^{r-1} y}{\mathrm{~d} t^{r-1}}\right)}{L_{g} L_{f}^{r-1} h\left(z_{1}, \ldots, z_{n-r}, y, \frac{\mathrm{d} y}{\mathrm{~d} t}, \ldots, \frac{\mathrm{d}^{r-1} y}{\mathrm{~d} t^{r-1}}\right)} .
\end{aligned}
$$

The unforced minimal-order inverse, i.e. the dynamic system

$$
\begin{aligned}
\dot{z}_{1} & =L_{f} t_{1}\left(z_{1}, \ldots, z_{n-r}, 0, \ldots, 0\right) \\
& \vdots \\
\dot{z}_{n-r} & =L_{f} t_{n-r}\left(z_{1}, \ldots, z_{n-r}, 0, \ldots, 0\right)
\end{aligned}
$$

is then called the (unforced) zero dynamics of the process, and provides a nonlinear analog of the con- 
cept of zeros in linear systems (Byrnes and Isidori, 1985). In analogy with the linear case, a process of the form of eq. (1) is said to be minimum phase if its zero dynamics [eq. (6)] is asymptotically stable.

\section{NONLINEAR PROCESS CONTROL: INPUT/OUTPUT VERSUS STATE-SPACE APPROACH}

In what follows we will provide a review of key elements of the input/output and state-space approaches in nonlinear process control. The review does not intend to be exhaustive; its primary purpose is to motivate the development that follows.

\section{Input/output approach}

Consider the classical error feedback control structure of Fig. 1, where $P$ and $C$ represent nonlinear input/output operators corresponding to the process and the classical feedback controller, respectively. In analogy with the linear case, consider also the $Q$ parametrization of the classical controller operator

$$
C=Q(I-P Q)^{-1}
$$

where $I$ denotes the identity operator and $Q$ an appropriate nonlinear operator. Then, one obtains the equivalent control structure of Fig. 2, which is usually referred to as the internal model control (IMC) structure, with $Q$ being the IMC controller. In the above setting, the input/output behavior of the closed-loop system is described by

$$
y=P C(I+P C)^{-1} y_{s p}
$$

or, equivalently, by

$$
y=P Q y_{s p}
$$

where $y_{s p}$ denotes the output set point. The controller synthesis problem can then be stated as follows: given the process operator $P$, specify the controller operator $C$ (or $Q$ ) that enforces a closed-loop response of the form

$$
\boldsymbol{y}=\boldsymbol{R} \boldsymbol{y}_{\mathrm{sp}}
$$

where $R$ is the desirable closed-loop operator (possibly nonlinear). The requirement of eq. (10) yields

$$
C=P^{-1} R(I-R)^{-1}
$$

or equivalently

$$
Q=P^{-1} R
$$

for the controller operators.

In the above framework, two issues of primary importance are the stability of the closed-loop system and the specification of the desired operator $R$. For the special case of a stable process operator $P$, it is clear that the closed-loop system will be stable if and only if the operator $\left(P^{-1} R\right)$ is stable. Therefore, if $P^{-1}$ is also stable (i.e. the process is minimum phase), one can choose $R$ to be any stable linear time-invariant operator. In the general case where the process operator $P$ can be unstable, the issue of closed-loop stability seems intractable in such an abstract setting. Even for the simplest case of open-loop stable minimumphase processes, however, the input/output approach cannot provide definitive answers to questions like:

(1) What order of $R$ would make the controller $C$ (or $Q$ ) proper?

(2) How should the controller $C$ (or $Q$ ) [eqs (11) and (12)] be implemented?

With respect to the first question, Economou et al. (1986) identified a connection between the order of $R$ and the concept of relative order, which arises rigorously from a state-space perspective. More

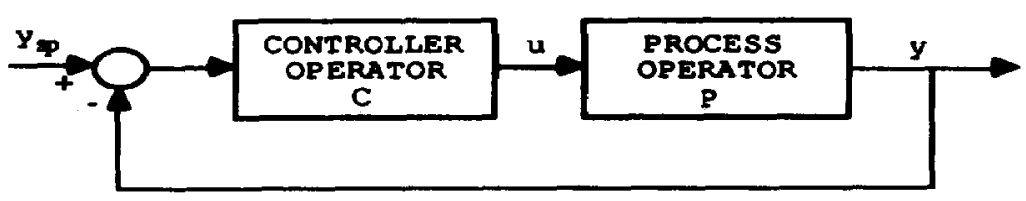

Fig. 1. Classical error feedback control structure.

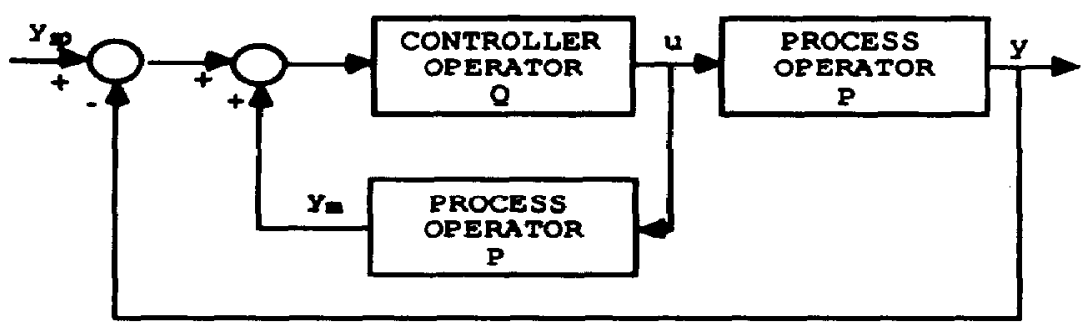

Fig. 2. IMC structure. 
specifically, since the inverse of the process requires differentiation of the output up to $r$ times [see eqs (2) and (5)], the operator $R$ must perform at least $r$ integrations to make the controller operator proper [see eqs (11) and (12)]. Consequently, the operator $R$ must be a dynamic system of relative order at least $r$. With respect to the second question, Economou et al. (1986) and Parrish and Brosilow (1988) proposed iterative numerical methods for the implementation of the inverse operator. Attempts of Economou et al. (1986) to use the Hirschorn inverse [eq. (2)] have failed, since, as can be easily verified, it suffers from internal instability due to pole-zero cancellations at the origin. As will be seen later, internally stable realizations of the inverse operator for the controller implementation can only be constructed in a state-space framework. In conclusion, although the input/output approach provides a macroscopic perspective and valuable insights on the role of the inverse operator in the controller synthesis problem, it cannot lead to explicit controller formulas, unless state-space considerations are also taken into account.

\section{State-space approach}

One of the most important controller synthesis results for minimum-phase nonlinear processes is the notion of input/output linearization, which we now briefly review. Referring to a minimum-phase nonlinear process of the form of eq. (1), with relative order $r$, the state feedback law (Kravaris and Chung, 1987)

$$
u=\frac{v-\sum_{k=0}^{r} \beta_{k} L_{f}^{k} h(x)}{\beta_{r} L_{g} L_{f}^{r-1} h(x)}
$$

induces the linear input/output behavior

$$
\sum_{k=0}^{r} \beta_{k} \frac{\mathrm{d}^{k} y}{\mathrm{~d} t^{k}}=v
$$

The input/output stability of the closed-loop system depends on the choice of the parameters $\beta_{k}$, while for an input/output stable closed-loop behavior, the internal stability of the closed-loop system is guaranteed by the minimum-phase assumption for the process. Once the input/output dynamics has been linearized, a linear controller can be used around the linear $(v-y)$ system, to incorporate integral action to the control structure. The resulting control structure is called the globally linearizing control (GLC) structure (Kravaris and Chung, 1987) and is shown in Fig. 3. A modification of the GLC structure has also been proposed for open-loop stable processes (Kravaris and Chung, 1987), where the state variables are reconstructed through an open-loop state observer, which is the process model itself. The resulting error feedback structure is depicted in Fig. 4. Note the similarity between this structure and the IMC structure of Fig. 2. The open-loop observer essentially acts as an internal model that is simulated in parallel to the process, while the input/output linearizing control law of eq. (13) can be interpreted as generating an internally stable inverse of the process model.

\section{DYNAMIC OUTPUT FEEDBACK CONTROL OF MINIMUM-PHASE NONLINEAR PROCESSES}

Referring to minimum-phase nonlinear processes of the form of eq. (1), we will now consider the problem of synthesizing a dynamic output feedback controller which induces a prespecified closed-loop response between the output $y$ and the set point $y_{s p}$. The assumption of minimum-phase behavior (i.e. stable zero dynamics) will allow requesting a closed-loop response of order $r$, resulting by cancelling the zero dynamics of the process. For convenience we will also request a linear closed-loop response, for which performance specifications can be easily expressed. The characterization "dynamic output feedback controller" implies that the controller will be a dynamic nonlinear system by itself, with inputs the set point

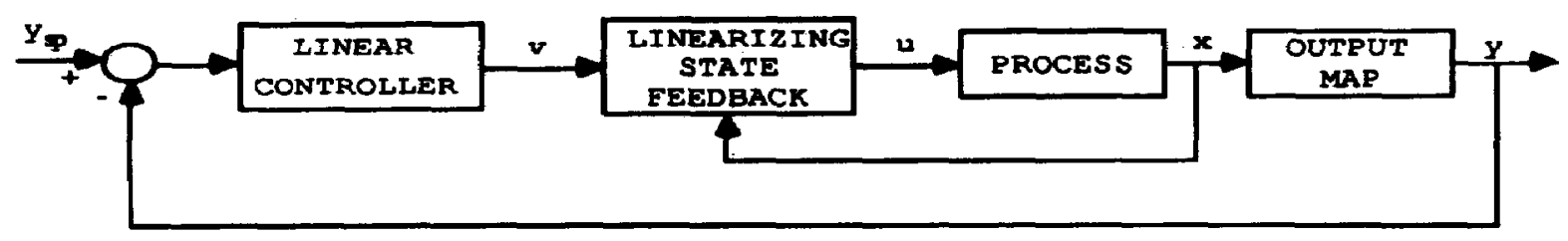

Fig. 3. GLC structure.

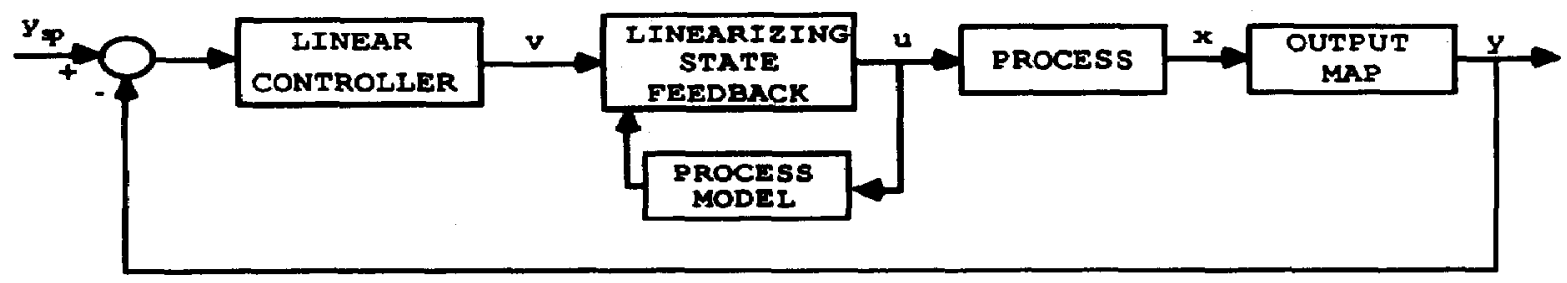

Fig. 4. GLC structure with open-loop state observer. 
$y_{s p}$ and the process output $y$ and output the process input $u$.

In what follows, the above synthesis problem will be addressed from a state-space perspective. In particular, given the state-space description of the process [eq. (1)], the problem will be to derive a state-space realization of a controller which will induce a closed-loop input/output behavior of the form

$$
y+\sum_{k=1}^{r} \gamma_{k} \frac{\mathrm{d}^{k} y}{\mathrm{~d} t^{k}}=y_{s p}
$$

where $\gamma_{k}$ are adjustable parameters. The derivation of the controller will essentially be based on combination of state feedback and state observers. The output feedback controllers that will be developed, will, however, find a transparent interpretation from an input/output perspective, establishing thus a precise connection between the two approaches.

Controller synthesis for open-loop stable nonlinear processes

The output feedback control problem for openloop stable processes can be handled through a GLC/open-loop observer configuration, as discussed in the previous section. The purpose of theorem 1 that follows is to state this result in a more precise and explicit way, allowing at the same time for alternative interpretations of the controller.

Theorem 1: Consider a nonlinear process of the form of eq. (1) with relative order $\mathrm{r}$. Then, the dynamic system

$$
\begin{aligned}
& \dot{\xi}=A^{*} \xi+b^{*}\left(y_{s p}-y\right) \\
& \dot{w}=f(w)+g(w) \frac{c^{*} \xi+\frac{\beta_{r}}{\gamma_{r}}\left(y_{s p}-y\right)-\sum_{k=0}^{r} \beta_{k} L_{f}^{k} h(w)}{\beta_{r} L_{g} L_{f}^{r-1} h(w)} \\
& u=\frac{c^{*} \xi+\frac{\beta_{r}}{\gamma_{r}}\left(y_{s p}-y\right)-\sum_{k=0}^{r} \beta_{k} L_{f}^{k} h(w)}{\beta_{r} L_{g} L_{f}^{r-1} h(w)}
\end{aligned}
$$

where $A^{*}, b^{*}, c^{*}$ are matrices of dimensions $r \times r, r \times 1$ and $1 \times \mathrm{r}$, respectively, given by: represents an $(\mathrm{n}+\mathrm{r})$ th order state-space realization of a dynamic output feedback controller which induces the closed-loop input/output behavior

$$
y+\sum_{k=1}^{r} \gamma_{k} \frac{\mathrm{d}^{k} y}{\mathrm{dt}^{k}}=y_{s p}
$$

Proof: Define the auxiliary variable

$$
v=c^{* \xi}+\frac{\beta_{r}}{\gamma_{r}}\left(y_{s p}-y\right) .
$$

Then, recalling standard linear systems theory, it can be easily verified that the system

$$
\begin{aligned}
& \xi=A^{*} \xi+b^{*}\left(y_{s p}-y\right) \\
& v=c^{*} \xi+\frac{\beta_{r}}{\gamma_{r}}\left(y_{s p}-y\right)
\end{aligned}
$$

represents a minimal state-space realization of

$$
\sum_{k=1}^{r} \gamma_{k} \frac{\mathrm{d}^{k} v}{\mathrm{~d} t^{k}}=\sum_{k=0}^{r} \beta_{k} \frac{\mathrm{d}^{k}\left(y_{s p}-y\right)}{\mathrm{d} t^{k}}
$$

with input the error $\left(y_{s p}-y\right)$ and output the auxiliary variable $v$, which can be interpreted as a choice of the linear controller in the GLC structure. The other component of the controller of eq. (16) becomes then

$$
\begin{aligned}
& \dot{w}=f(w)+g(w) \frac{v-\sum_{k=0}^{r} \beta_{k} L_{f}^{k} h(w)}{\beta_{r} L_{g} L_{f}^{-1} h(w)} \\
& u=\frac{v-\sum_{k=0}^{r} \beta_{k} L_{f}^{k} h(w)}{\beta_{r} L_{g} L_{f}^{r-1} h(w)}
\end{aligned}
$$

which is an input/output linearizing state feedback law, with the states reconstructed through an openloop observer. Under consistent initialization of $w$ and $x$ [i.e. $w(0)=x(0)]$, it easily follows that $w(t)=x(t)$; then, eq. (21) induces exactly the behavior of eq. (14)

$$
\sum_{k=0}^{\infty} \beta_{k} \frac{\mathrm{d}^{k} y}{\mathrm{~d} t^{k}}=v
$$

$$
\begin{aligned}
& A^{*}=\left[\begin{array}{cccccc}
0 & 1 & 0 & \cdots & 0 & 0 \\
0 & 0 & 1 & \cdots & 0 & 0 \\
0 & 0 & 0 & \cdots & 0 & 0 \\
\vdots & \vdots & \vdots & \ddots & \vdots & \vdots \\
0 & 0 & 0 & \cdots & 0 & 1 \\
0 & -\frac{\gamma_{1}}{\gamma_{r}} & -\frac{\gamma_{2}}{\gamma_{r}} & \cdots & -\frac{\gamma_{r-2}}{\gamma_{r}} & -\frac{\gamma_{r-1}}{\gamma_{r}}
\end{array}\right], \quad b^{*}=\left[\begin{array}{c}
0 \\
0 \\
0 \\
\vdots \\
0 \\
\frac{1}{\gamma_{r}}
\end{array}\right],
\end{aligned}
$$

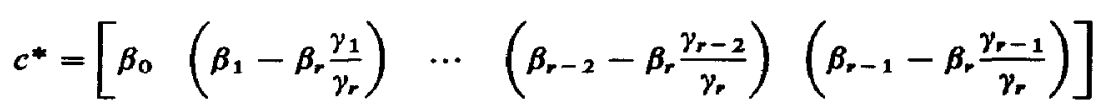


Combining eq. (14) and eq. (20) we easily obtain the desired closed-loop input/output dynamics:

$$
y+\sum_{k=1}^{r} \gamma_{k} \frac{\mathrm{d}^{k} y}{\mathrm{~d} t^{k}}=y_{s p}
$$

From an input/output perspective, the dynamic controller of theorem 1 is clearly a one-degree-offreedom controller and in particular an error feedback controller; its input is the error $\left(y_{s p}-y\right)$ and its output is the value of the process input $u$, while it involves $(n+r)$ state variables. Therefore, the control structure resulting from theorem 1 coincides with the classical error feedback control structure of Fig. 1, and eq. (16) can be interpreted as a state-space realization of the classical controller operator $C$.

Furthermore, referring to eq. (11), one can easily identify two distinct components of the controller. The first component is the dynamic system

$$
\begin{aligned}
\xi & =A^{*} \xi+b^{*}\left(y_{s p}-y\right) \\
y^{*} & =\xi_{1}
\end{aligned}
$$

which represents a minimal state-space realization of the input/output dynamics described by the operator

$$
R(I-R)^{-1}=\left(\sum_{k=1}^{r} \gamma_{k} \frac{\mathrm{d}^{k}}{\mathrm{~d} t^{k}}\right)^{-1}
$$

with input the error $\left(y_{s p}-y\right)$ and output the auxiliary variable $y^{*}$. From eq. (22) it can be easily verified that

$$
c^{*} \xi+\frac{\beta_{r}}{\gamma_{r}}\left(y_{s p}-y\right)=\sum_{k=0}^{r} \beta_{k} \frac{d^{k} y^{*}}{d t^{k}} .
$$

Then, the second component of the controller takes the form

$$
\begin{aligned}
& \dot{w}=f(w)+g(w) \frac{\sum_{k=0}^{r} \beta_{k} \frac{d^{k} y^{*}}{d t^{k}}-\sum_{k=0}^{r} \beta_{k} L_{f}^{k} h(w)}{\beta_{r} L_{g} L_{f}^{r-1} h(w)} \\
& u=\frac{\sum_{k=0}^{r} \beta_{k} \frac{d^{k} y^{*}}{d t^{k}}-\sum_{k=0}^{r} \beta_{k} L_{f}^{k} h(w)}{\beta_{r} L_{k} L_{f}^{r-1} h(w)}
\end{aligned}
$$

which is an internally stable (full-order) realization of the process inverse operator $P^{-1}$, in the sense that when driven by $y^{*}$, it produces the process input $u$ necessary to make the process output $y$ equal to $y^{*}$. The two components of the controller identified above are shown explicitly in Fig. 5.

Remark 1 (reduced-order realization): It is interesting to observe that the realization of the controller of eq. (16) is not minimal. Indeed, from the second equation of eq. (16) it easily follows that

$$
\sum_{k=0}^{r} \beta_{k} \frac{\mathrm{d}^{k}[h(w)]}{\mathrm{d} t^{k}}=c^{*} \xi+\frac{\beta_{r}}{\gamma_{r}}\left(y_{s p}-y\right)
$$

which combined with eq. (24) yields

Thus, if

$$
\sum_{k=0}^{r} \beta_{k} \frac{\mathrm{d}^{k}[h(w)]}{\mathrm{d} t^{k}}=\sum_{k=0}^{r} \beta_{k} \frac{\mathrm{d}^{k} y^{*}}{\mathrm{~d} t^{k}}
$$

$$
\xi_{i}(0)=L_{f}^{i-1} h(w)(0), \quad i=1, \ldots, r
$$

it follows that

$$
\xi_{i}=L_{f}^{i-1} h(w), \quad i=1, \ldots, r
$$

Substituting the relations of eq. (29) into the second and third equations of eq. (16), we easily obtain the following $n$th order realization of the controller

$$
\begin{aligned}
w & =f(w)+g(w) \frac{\left(y_{s p}-y\right)-\sum_{k=1}^{r} \gamma_{k} L_{f}^{k} h(w)}{\gamma_{r} L_{\theta} L_{f}^{r-1} h(w)} \\
u & =\frac{\left(y_{s p}-y\right)-\sum_{k=1}^{r} \gamma_{k} L_{f}^{k} h(w)}{\gamma_{r} L_{g} L_{f}^{r-1} h(w)} .
\end{aligned}
$$

Equation (30) can be interpreted as a feedforward controller on the error $\left(y_{s p}-y\right)$, which enforces the dynamics

$$
\sum_{k=1}^{r} \gamma_{k} \frac{\mathrm{d}^{k} y}{\mathrm{~d} t^{k}}=\left(y_{s p}-y\right)
$$

between the error $\left(y_{s p}-y\right)$ and the process output $y$.

Remark 2 (IMC realization): The reduced-order controller realization of eq. (30) is equivalent to the realization

$$
\begin{aligned}
\dot{w}= & f(w)+g(w) \\
& \times \frac{\left(y_{s p}-y+y_{m}\right)-h(w)-\sum_{k=1}^{r} \gamma_{k} L_{f}^{k} h(w)}{\gamma_{r} L_{g} L_{f}^{r-1} h(w)} \\
u & =\frac{\left(y_{s p}-y+y_{m}\right)-h(w)-\sum_{k=1}^{r} \gamma_{k} L_{f}^{k} h(w)}{\gamma_{r} L_{g} L_{f}^{r-1} h(w)} \\
\dot{x}_{m} & =f\left(x_{m}\right)+g\left(x_{m}\right) u \\
y_{m} & =h\left(x_{m}\right)
\end{aligned}
$$

under consistent initialization of $w$ and $x_{m}\left[w(0)=x_{m}(0)\right.$ clearly implies that $w(t)=x_{m}(t)$ and $\left.y_{m}=h(w)\right]$.

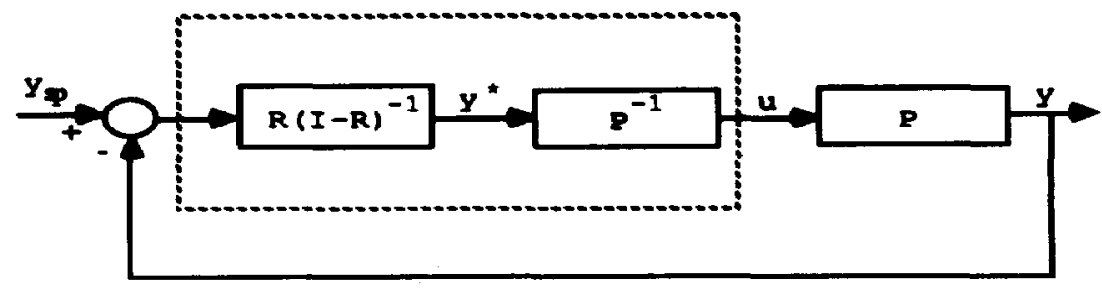

Fig. 5. Error feedback control structure. 
Equation (32) represents a reduced-order IMC realization of the controller (see Fig. 2), consisting of realizations of the controller operator $Q$ and the process model $P$. A similar realization was proposed by Henson and Seborg (1991), where the controller operator $Q$ was interpreted as an internally unstable realization of the process inverse combined with a nonlinear filter. Finally, from eq. (32) and using similar arguments as in remark 1 , one easily obtains a fullorder IMC realization of the controller, given by: and output $u$. Finally, the remaining $n$ equations represent a realization of the process model $P$. The components of the controller identified above are shown explicitly in Fig. 6.

Controller synthesis for general nonlinear processes

The applicability of the output feedback controller of theorem 1 and the control structure of Fig. 5 is

$\dot{\xi}=\left[\begin{array}{cccccc}0 & 1 & 0 & \cdots & 0 & 0 \\ 0 & 0 & 1 & \cdots & 0 & 0 \\ 0 & 0 & 0 & \cdots & 0 & 0 \\ \vdots & \vdots & \vdots & \ddots & \vdots & \vdots \\ 0 & 0 & 0 & \cdots & 0 & 1 \\ -\frac{1}{\gamma_{r}} & -\frac{\gamma_{1}}{\gamma_{r}} & -\frac{\gamma_{2}}{\gamma_{r}} & \cdots & -\frac{\gamma_{r-2}}{\gamma_{r}} & -\frac{\gamma_{r-1}}{\gamma_{r}}\end{array}\right] \xi+\left[\begin{array}{c}0 \\ 0 \\ 0 \\ \vdots \\ 0 \\ \frac{1}{\gamma_{r}}\end{array}\right]\left(y_{s p}-y+y_{m}\right)$

$\dot{w}=f(w)+g(w)$

$$
\times \frac{\left(\beta_{0}-\beta_{r} \frac{1}{\gamma_{r}}\right) \xi_{1}+\left(\beta_{1}-\beta_{r} \frac{\gamma_{1}}{\gamma_{r}}\right) \xi_{2}+\cdots+\left(\beta_{r-1}-\beta_{r} \frac{\gamma_{r-1}}{\gamma_{r}}\right) \xi_{r}+\frac{\beta_{r}}{\gamma_{r}}\left(y_{s p}-y+y_{m}\right)-\sum_{k=0}^{r} \beta_{k} L_{f}^{k} h(w)}{\gamma_{r} L_{a} L_{f}^{r-1} h(w)}
$$

$u=\frac{\left(\beta_{0}-\beta_{r} \frac{1}{\gamma_{r}}\right) \xi_{1}+\left(\beta_{1}-\beta_{r} \frac{\gamma_{1}}{\gamma_{r}}\right) \xi_{2}+\cdots+\left(\beta_{r-1}-\beta_{r} \frac{\gamma_{r-1}}{\gamma_{r}}\right) \xi_{r}+\frac{\beta_{r}}{\gamma_{r}}\left(y_{s p}-y+y_{m}\right)-\sum_{k=0}^{r} \beta_{k} L_{f}^{k} h(w)}{\gamma_{r} L_{g} L_{f}^{r-1} h(w)}$

$\dot{x}_{m}=f\left(x_{m}\right)+g\left(x_{m}\right) u$

$y_{m}=h\left(x_{m}\right)$.

The above realization allows identifying directly the various components of the controller. In particular, the first $r$ equations of eq. (33) represent a realization of the operator $R$ (called "filter" in the IMC terminology), with input $\left(y_{s_{p}}-y+y_{m}\right)$ and output $y^{*}=\xi_{1}$. The next $n$ equations represent a realization of the process inverse $P^{-1}$, with input

$$
\begin{aligned}
& \left(\beta_{0}-\beta_{r} \frac{1}{\gamma_{r}}\right) \xi_{1}+\left(\beta_{1}-\beta_{r} \frac{\gamma_{1}}{\gamma_{r}}\right) \xi_{2}+\cdots \\
& \quad+\left(\beta_{r-1}-\beta_{r} \frac{\gamma_{r-1}}{\gamma_{r}}\right) \xi_{r} \\
& \quad+\frac{\beta_{r}}{\gamma_{r}}\left(y_{s p}-y+y_{m}\right)=\sum_{k=0}^{r} \beta_{k} \frac{\mathrm{d}^{k} y^{*}}{\mathrm{~d} t^{k}}
\end{aligned}
$$

limited by the assumption of open-loop stability for the process. This occurs for the following reasons:

- The controller of theorem 1 incorporates an open-loop state observer, which is the process model itself. Therefore, in the case of an openloop unstable process, any error in the initial conditions will grow indefinitely.

- The control structure of Fig. 5 involves a series connection of $P$ and $P^{-1}$. Therefore, in the case of an open-loop unstable process, it will essentially involve cancellation of unstable dynamics, with obvious consequences on the internal stability of the closed-loop system.

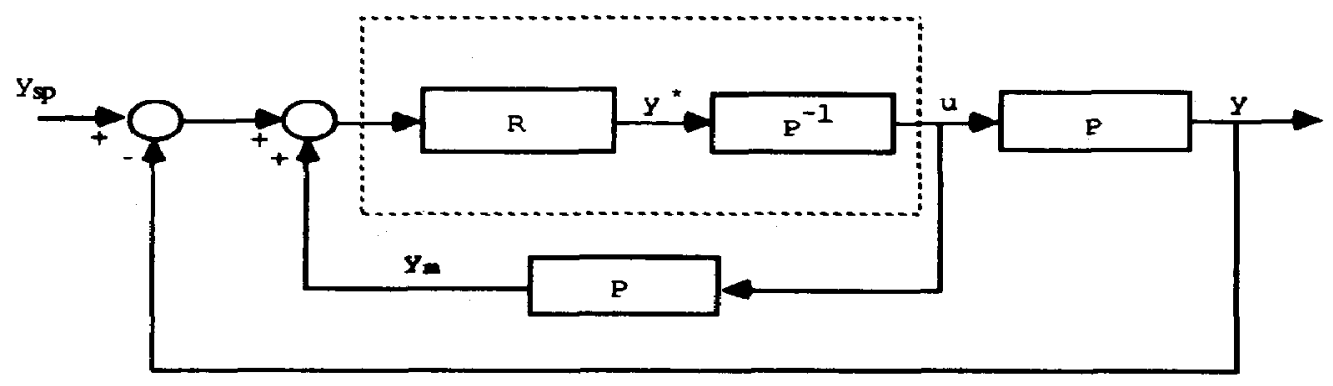

Fig. 6. IMC form of the error feedback control structure. 
It is apparent from the above, that, in order to cope with a potential instability of the process, a different kind of observer must be used together with an appropriate modification of the control structure. To this end, the normal form representation of the process is of great convenience. In particular, for minimumphase processes, and referring to their normal form representation [eq. (4)], the $(n-r)$ states of the zero
- an error feedback component

$$
\begin{aligned}
& \xi=A^{*} \xi+b^{*}\left(y_{s p}-y\right) \\
& v=c^{*} \xi+\frac{\beta_{r}}{\gamma_{r}}\left(y_{s p}-y\right)
\end{aligned}
$$

which can be interpreted as a choice of the linear controller of the GLC structure with input/output dynamics given by eq. (20) and

- an output feedback component

$$
\begin{gathered}
{\left[\begin{array}{c}
\dot{z}_{1} \\
\vdots \\
\dot{z}_{n-r}
\end{array}\right]=\left[\begin{array}{c}
L_{f} t_{1}\left(z_{1}, \ldots, z_{n-r}, y, \frac{\mathrm{d} y}{\mathrm{~d} t}, \ldots, \frac{\mathrm{d}^{r-1} y}{\mathrm{~d} t^{r-1}}\right) \\
\vdots \\
L_{f} t_{n-r}\left(z_{1}, \ldots, z_{n-r}, y, \frac{\mathrm{d} y}{\mathrm{~d} t}, \ldots, \frac{\mathrm{d}^{r-1} y}{\mathrm{~d} t^{r-1}}\right)
\end{array}\right]} \\
u=\frac{v-\sum_{k=0}^{r-1} \beta_{k} \frac{\mathrm{d}^{k} y}{\mathrm{~d} t^{k}}-\beta_{r} L_{f}^{r} h\left(z_{1}, \ldots, z_{n-r}, y, \frac{\mathrm{d} y}{\mathrm{~d} t}, \ldots, \frac{\mathrm{d}^{r-1} y}{\mathrm{~d} t^{r-1}}\right)}{\beta_{r} L_{g} L_{f}^{r-1} h\left(z_{1}, \ldots, z_{n-r}, y, \frac{\mathrm{d} y}{\mathrm{~d} t}, \ldots, \frac{\mathrm{d}^{r-1} y}{\mathrm{~d} t^{r-1}}\right)}
\end{gathered}
$$

dynamics can be reconstructed by using measurements of the output and its derivatives. The remaining $r$ state variables are exactly the output and its derivatives up to $(r-1)$ th order, assumed available. Theorem 2 that follows provides a solution to the general output feedback synthesis problem for minimum-phase systems, along the above lines. For convenience, the controller is written in the normal form coordinates.

Theorem 2: Consider a nonlinear process with relative order $r$, in its normal form description of eq. (4). Then, the dynamic system:

$$
\begin{gathered}
{\left[\begin{array}{c}
\dot{z}_{1} \\
\vdots \\
\dot{z}_{n-r}
\end{array}\right]=\left[\begin{array}{c}
L_{f} t_{1}\left(z_{1}, \ldots, z_{n-r}, y, \frac{\mathrm{d} y}{\mathrm{~d} t}, \ldots, \frac{\mathrm{d}^{r-1} y}{\mathrm{~d} t^{r-1}}\right) \\
\vdots \\
L_{f} t_{n-r}\left(z_{1}, \ldots, z_{n-r}, y, \frac{\mathrm{d} y}{\mathrm{~d} t}, \ldots, \frac{\mathrm{d}^{r-1} y}{\mathrm{~d} t^{r-1}}\right)
\end{array}\right]} \\
u=\frac{c^{*} \xi+\frac{\beta_{r}}{\gamma_{r}}\left(y_{s p}-y\right)-\sum_{k=0}^{r-1} \beta_{k} \frac{\mathrm{d}^{k} y}{\mathrm{~d} t^{k}}-\beta_{r} L_{f}^{r} h\left(z_{1}, \ldots, z_{n-r}, y, \frac{\mathrm{d} y}{\mathrm{~d} t}, \ldots, \frac{\mathrm{d}^{r-1} y}{\mathrm{~d} t^{r-1}}\right)}{\beta_{r} L_{g} L_{f}^{r-1} h\left(z_{1}, \ldots, z_{n-r}, y, \frac{\mathrm{d} y}{\mathrm{~d} t}, \ldots, \frac{\mathrm{d}^{r-1} y}{\mathrm{~d} t^{r-1}}\right)}
\end{gathered}
$$

where $\mathrm{A}^{*}, \mathrm{~b}^{*}, \mathrm{c}^{*}$ are given by eq. (17), represents an $\mathrm{nth}$ order state-space realization of a dynamic output feedback controller which induces the closed-loop input/output behavior

$$
y+\sum_{k=1}^{r} \gamma_{k} \frac{\mathrm{d}^{k} y}{\mathrm{~d} t^{k}}=y_{s p}
$$

Proof: The proof outline is the same as the one in theorem 1. In particular, the controller of eq. (34) is composed of: which is essentially an input/output linearizing state feedback law, which makes explicit use of the output and its derivatives up to $(r-1)$ th order, while the other $(n-r)$ states are reconstructed through a reduced-order observer.

Under consistent initialization of $z$ in eq. (35) and $\zeta$ in the process normal form of eq. (4), i.e. $\zeta_{l}(0)=z_{i}(0)$, $i=1, \ldots,(n-r)$, it easily follows that $\zeta_{i}(t)=z_{i}(t)$, $i=1, \ldots,(n-r)$. Then, given that $\zeta_{n-r+j}=$ $\mathrm{d}^{j-1} y / \mathrm{d} t^{j-1}, j=1, \ldots, r$, eq. (35) induces exactly the dynamics of eq. (14); combining eq. (14) with eq. (20) results in the desired closed-loop input/output behavior.

Clearly, the controller of theorem 2 is a nonlinear analogue of a two-degree-of-freedom controller, i.e. a mixed error and output feedback controller, with the two controller blocks shown explicitly in Fig. 7. This is in agreement with the intuition from linear systems, where a two-degree-of-freedom controller is 
usually employed for open-loop unstable systems, with the output feedback essentially having a stabilizing effect on the overall structure. It is conceivable that a similar control structure can be obtained in an IMC framework, with an output feedback controller employed in an inner loop to stabilize the unstable process (Economou et al., 1986).

In analogy with the treatment in the previous subsection, the controller of eq. (34) can find an input/output interpretation, as follows. The error
The latter equation can be loosely interpreted as a minimal-order realization of the process inverse operator $P^{-1}$, in the sense that when driven by $y^{*}$ and $y$, it produces the process input $u$ necessary to make the process output $y$ equal to $y^{*}$. Figure 8 identifies explicitly the above input/output operators. A comparison with Fig. 5 shows the modified control structure which accounts for possible open-loop instability.

Remark 3: In the special case of $r=1$, the controller of eq. (34) reduces to

$$
\begin{aligned}
\dot{\xi}_{1} & =\frac{1}{\gamma_{1}}\left(y_{s p}-y\right) \\
{\left[\begin{array}{c}
\dot{z}_{1} \\
\vdots \\
\dot{z}_{n-1}
\end{array}\right]=} & {\left[\begin{array}{c}
L_{f} t_{1}\left(z_{1}, \ldots, z_{n-1}, y\right) \\
\vdots \\
L_{f} t_{n-1}\left(z_{1}, \ldots, z_{m-1}, y\right)
\end{array}\right] } \\
u & =\frac{\beta_{0} \xi_{1}+\frac{\beta_{1}}{\gamma_{1}}\left(y_{s p}-y\right)-\left[\beta_{0} y+\beta_{1} L_{f} h\left(z_{1}, \ldots, z_{n-1}, y\right)\right]}{\beta_{1} L_{g} h\left(z_{1}, \ldots, z_{n-1}, y\right)}
\end{aligned}
$$

or equivalently to

$$
\begin{aligned}
{\left[\begin{array}{c}
\dot{z}_{1} \\
\vdots \\
\dot{z}_{n-1}
\end{array}\right]=\left[\begin{array}{c}
L_{f} t_{1}\left(z_{1}, \ldots, z_{n-1}, y\right) \\
\vdots \\
L_{f} t_{n-1}\left(z_{1}, \ldots, z_{n-1}, y\right)
\end{array}\right] } \\
u=\frac{\frac{1}{\gamma_{1}}\left[\left(y_{s p}-y\right)+\frac{\beta_{0}}{\beta_{1}} \int_{0}^{t}\left(y_{s p}-y\right) \mathrm{d} t\right]-\left[\frac{\beta_{0}}{\beta_{1}} y+L_{f} h\left(z_{1}, \ldots, z_{n-1}, y\right)\right]}{L_{g} h\left(z_{1}, \ldots, z_{n-1}, y\right)} .
\end{aligned}
$$

feedback component of the controller can be written in the form of eq. (22), which is a minimal state-space realization of the operator $R(I-R)^{-1}$. Then, given eq. (24), the output feedback component of the controller takes the form
The error feedback part of the controller is a PI controller with settings $K_{c}=1 / \gamma_{1}$ and $\tau_{I}=\beta_{1} / \beta_{0}$, while the output feedback part of the controller uses measurements of the process output $y$ only.

Remark 4: In the special case of $r=n$, the controller of eq. (34) takes the form

$$
\begin{aligned}
{\left[\begin{array}{c}
z_{1} \\
\vdots \\
\dot{z}_{n-r}
\end{array}\right]=\left[\begin{array}{c}
L_{f} t_{1}\left(z_{1}, \ldots, z_{n-r}, y, \frac{\mathrm{d} y}{\mathrm{~d} t}, \ldots, \frac{\mathrm{d}^{r-1} y}{\mathrm{~d} t^{r-1}}\right) \\
\vdots \\
L_{f} t_{n-r}\left(z_{1}, \ldots, z_{n-r}, y, \frac{\mathrm{d} y}{\mathrm{~d} t}, \ldots, \frac{\mathrm{d}^{r-1} y}{\mathrm{~d} t^{r-1}}\right)
\end{array}\right] } \\
u=\frac{\sum_{k=0}^{r} \beta_{k} \frac{\mathrm{d}^{k} y^{*}}{\mathrm{~d} t^{k}}-\sum_{k=0}^{r-1} \beta_{k} \frac{\mathrm{d}^{k} y}{\mathrm{~d} t^{k}}-\beta_{r} L_{f}^{r} h\left(z_{1}, \ldots, z_{n-r}, y, \frac{\mathrm{d} y}{\mathrm{~d} t}, \ldots, \frac{\mathrm{d}^{r-1} y}{\mathrm{~d} t^{r-1}}\right)}{\beta_{r} L_{g} L_{f}^{r-1} h\left(z_{1}, \ldots, z_{n-r}, y, \frac{\mathrm{d} y}{\mathrm{~d} t}, \ldots, \frac{\mathrm{d}^{r-1} y}{\mathrm{~d} t^{r-1}}\right)} .
\end{aligned}
$$

$$
\begin{aligned}
& \xi=A^{*} \xi+b^{*}\left(y_{s p}-y\right) \\
& u=\frac{c^{*} \xi+\frac{\beta_{n}}{\gamma_{n}}\left(y_{s p}-y\right)-\sum_{k=0}^{n-1} \beta_{k} \frac{\mathrm{d}^{k} y}{\mathrm{~d} t^{k}}-\beta_{n} L_{f}^{n} h\left(y, \frac{\mathrm{d} y}{\mathrm{~d} t}, \ldots, \frac{\mathrm{d}^{n-1} y}{\mathrm{~d} t^{n-1}}\right)}{\beta_{n} L_{g} L_{j}^{n-1} h\left(y, \frac{\mathrm{d} y}{\mathrm{~d} t}, \ldots, \frac{\mathrm{d}^{n-1} y}{\mathrm{~d} t^{n-1}}\right)} .
\end{aligned}
$$




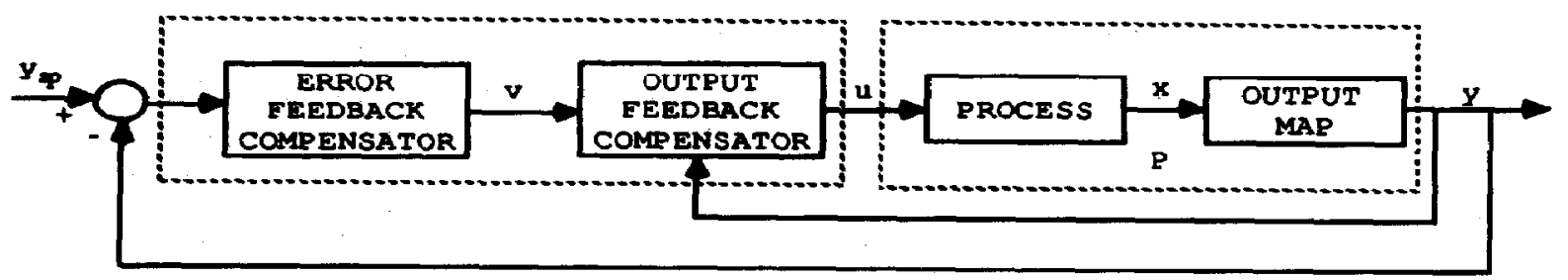

Fig. 7. Error and output feedback control structure (a).

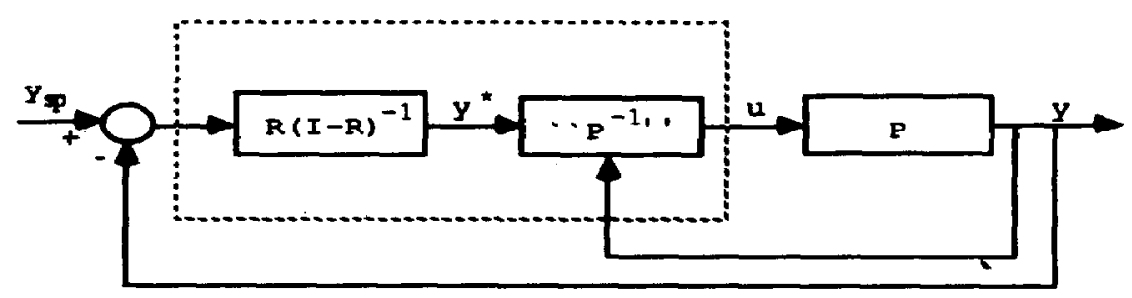

Fig. 8. Error and output feedback control structure (b).

This case includes a class of input/output models which have been considered in the context of the reference system synthesis control methodology (Bartee et al., 1989), i.e. quasi-linear ODE models of the form

$$
\frac{\mathrm{d}^{n} y}{\mathrm{~d} t^{n}}+a_{1}(y) \frac{\mathrm{d}^{n-1} y}{\mathrm{~d} t^{n-1}}+\cdots+a_{n-1}(y) \frac{\mathrm{d} y}{\mathrm{~d} t}+a_{n}(y)=u \text {. }
$$

Defining $y$ and its derivatives up to $(n-1)$ th order as state variables, such models can be represented as state-space models of the form

$$
\begin{aligned}
\dot{x}_{1}= & x_{2} \\
\dot{x}_{2}= & x_{3} \\
\vdots & \vdots \\
\dot{x}_{n-1}= & x_{n} \\
\dot{x}_{n}= & -a_{1}\left(x_{1}\right) x_{n-1}-\cdots-a_{n-1}\left(x_{1}\right) x_{2} \\
& \quad-a_{n}\left(x_{1}\right)+u \\
y= & x_{1}
\end{aligned}
$$

for which clearly $r=n$, and eq. (39) is applicable under appropriate definition of $f, g, h$.

Remark 5: In order to achieve a critically damped closed-loop response of the form:

$$
\left(1+\lambda \frac{\mathrm{d}}{\mathrm{d} t}\right)^{r} y=y_{s p}
$$

one simply uses the controller formulas of theorems 1 or 2 , with the particular choice of the parameters

$$
\gamma_{k}=\left(\begin{array}{l}
r \\
k
\end{array}\right) \lambda^{k}, \quad k=1, \ldots, r .
$$

Remark 6: Theorems 1 and 2 are very similar in philosophy. They essentially utilize different internally stable state-space realizations of the process inverse as part of the controller. Theorem 1 uses a full-order realization, while theorem 2 uses a minimal realization (with measurements of the output and its derivatives). Of course, theorem 1 is applicable only to open-loop stable processes, while theorem 2 is applicable to open-loop unstable processes as well. Furthermore, the implementation of the controller of theorem 2 for $r \geqslant 3$ may require filtering of the output signal or approximation of the output derivatives, in order to suppress noise effects. Therefore, the best choice of an output feedback controller for a particular process will be determined by the characteristics of the process itself.

\section{Closed-loop stability}

In the control structures resulting from theorems 1 and 2, the input/output stability of the closed-loop system will be guaranteed by a choice of the parameters $\gamma_{k}$ such that the roots of the characteristic equation

$$
1+\gamma_{1} s+\cdots+\gamma_{r} s=0
$$

lie in the left half of the complex plane. In addition to input/output stability, one must obtain a characterization for the internal stability of the closed-loop system, i.e. the asymptotic stability of the states in the unforced closed-loop system, for perturbations in the initial conditions. Employing a standard linear stability analysis, it is straightforward to show that the unforced closed-loop system $\left(y_{s_{p}}=0\right)$ under the controller of eq. (16) is locally asymptotically stable, if the following conditions are satisfied: 
(1) The open-loop process is locally exponentially stable.

(2) The roots of the characteristic equation

$$
\beta_{0}+\beta_{1} s+\cdots+\beta_{r} s^{r}=0
$$

lie in the left half of the complex plane.

(3) The zero dynamics of the process is locally exponentially stable. ture was employed using Newton's method for the calculation of the control law. The purpose of this study is to illustrate the application of the derived analytical controllers to the same process.

Setting $x_{1}=c_{A}, x_{2}=c_{B}, x_{3}=T, u=T_{0}$ and $y=c_{B} / c_{A}+c_{B}$, the dynamic model of the process can be easily put in the form of eq. (1), with

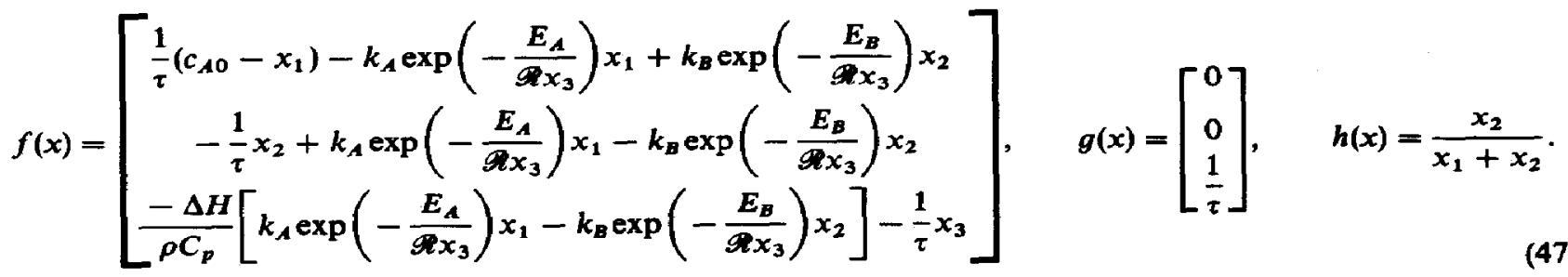

In a similar way, one can show that the unforced closed-loop system under the controller of eq. (34) will be locally asymptotically stable if conditions 2 and 3 from above are satisfied.

\section{EXAMPLE}

Consider the ideal continuous stirred tank reactor (CSTR) shown in Fig. 9. A solution stream consisting of $A$ at concentration $c_{\mathcal{A}}$ and temperature $T_{0}$ enters the reactor, where the reversible exothermic reaction

\section{$\mathrm{A} \rightleftarrows \mathrm{B}$}

takes place. The residence time in the reactor is $\tau$, assumed constant. The effluent stream leaves the reactor at concentrations $c_{A}, c_{B}$ and temperature $T$. Under standard assumptions, the mass and energy balances describing the dynamic behavior of the process take the form

$$
\begin{aligned}
& \frac{\mathrm{d} c_{A}}{\mathrm{~d} t}=\frac{1}{\tau}\left(c_{A 0}-c_{A}\right)-k_{A} \exp \left(-\frac{E_{A}}{\mathscr{R} T}\right) c_{A} \\
& +k_{B} \exp \left(-\frac{E_{B}}{\mathscr{S T} T}\right) c_{B} \\
& \frac{\mathrm{d} c_{B}}{\mathrm{~d} t}=-\frac{1}{\tau} c_{B}+k_{A} \exp \left(-\frac{E_{A}}{\operatorname{RT} T}\right) c_{A} \\
& -k_{\mathrm{B}} \exp \left(-\frac{E_{B}}{S T}\right) c_{\mathrm{B}} \\
& \frac{\mathrm{d} T}{\mathrm{~d} t}=\frac{-\Delta H}{\rho C_{p}}\left[k_{A} \exp \left(-\frac{E_{A}}{S T T}\right) c_{A}\right. \\
& \left.-k_{B} \exp \left(-\frac{E_{B}}{\mathscr{X} T}\right) c_{B}\right]+\frac{1}{\tau}\left(T_{0}-T\right)
\end{aligned}
$$

where the values of the various process parameters are shown in Table 1 . The control objective is to operate the reactor at a desired conversion, by manipulating the inlet temperature $T_{0}$. The above process was initially studied by Economou and Morari (1985) and Economou et al. (1986), where a nonlinear IMC struc-
The relative order of the output $y$ with respect to the manipulated input $u$ is easily found to be $r=2$, since $L_{g} L_{f} h(x)=\left(k_{A} E_{A} / \mathscr{S} x_{3}^{2}\right) \exp \left(-E_{A} / g x_{3}\right) x_{1}-$ $\left(k_{B} E_{B} / A x_{3}^{2}\right) \exp \left(-E_{B} / \mathscr{A} x_{3}\right) x_{2} \neq 0$. The set of points in state space for which $L_{f} L_{f} h(x)=0$ correspond to a singular surface, where the relative order is not well-defined and invertibility is lost. Figure 10 shows the conversion versus temperature equilibrium diagram and the singular line for the particular process (note that, since $x_{1}+x_{2}=c_{A 0}$, assuming constant inlet concentration effectively reduces the problem to a two-dimensional one). It can be easily seen that the equilibrium diagram has a well-defined maximum, while the singular line crosses the equilibrium line exactly at the point of maximum conversion. For this reason, operation of the reactor exactly at the maximum conversion is not feasible. For this particular study, the control objective was to operate the reactor at a conversion $y=0.508$, with the maximum conversion being $y=0.5087$ for an inlet temperature $u=435 \mathrm{~K}$. Because of the open-loop stability of the process, the controller realization of eq. (16) was employed. The adjustable parameters were chosen as $\beta_{0}=1, \beta_{1}=\gamma_{1}=120$ and $\beta_{2}=\gamma_{2}=3600$, in order to obtain a critically damped closed-loop response with time constant equal to $60 \mathrm{~s}$. Two representative runs of the reactor are presented. In the first run, the reactor is initially assumed to be at the steady state $x_{1 s}=0.84 \mathrm{gmoll}^{-1}, x_{2 s}=0.16 \mathrm{gmol}^{-1}$ and $x_{3 s}=353 \mathrm{~K}$, which corresponds to a point at the left

E $\mathbf{T}_{0}, \mathrm{C}_{\mathrm{AO}}$

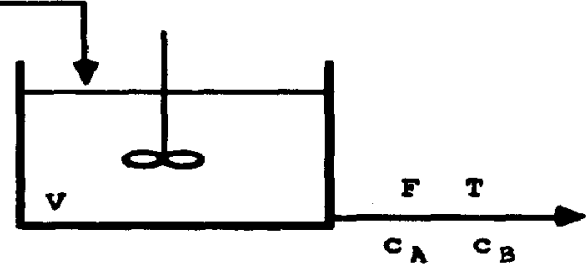

Fig. 9. A continuous stirred tank reactor (CSTR). 
Table 1. Process parameters

\begin{tabular}{rlrl}
$\tau$ & $=60 \mathrm{~s}$ & $g$ & $=1.987 \mathrm{cal} \mathrm{gmol}^{-1} \mathrm{~K}^{-1}$ \\
$k_{A}$ & $=5 \times 10^{3} \mathrm{~s}^{-1}$ & $-\Delta H$ & $=5 \times 10^{3} \mathrm{cal} \mathrm{gmol}^{-1}$ \\
$k_{B}$ & $=1 \times 10^{6} \mathrm{~s}^{-1}$ & $\rho$ & $=1 \mathrm{~kg} \mathrm{l}^{-1}$ \\
$E_{A}$ & $=1 \times 10^{4} \mathrm{cal} \mathrm{gmol}^{-1}$ & $C_{p}$ & $=1 \times 10^{3} \mathrm{cal} \mathrm{kg}^{-1} \mathrm{~K}^{-1}$ \\
$E_{B}$ & $=1.5 \times 10^{4} \mathrm{cal} \mathrm{gmol}^{-1}$ & $c_{A 0}$ & $=1 \mathrm{gmoll}^{-1}$ \\
\hline
\end{tabular}

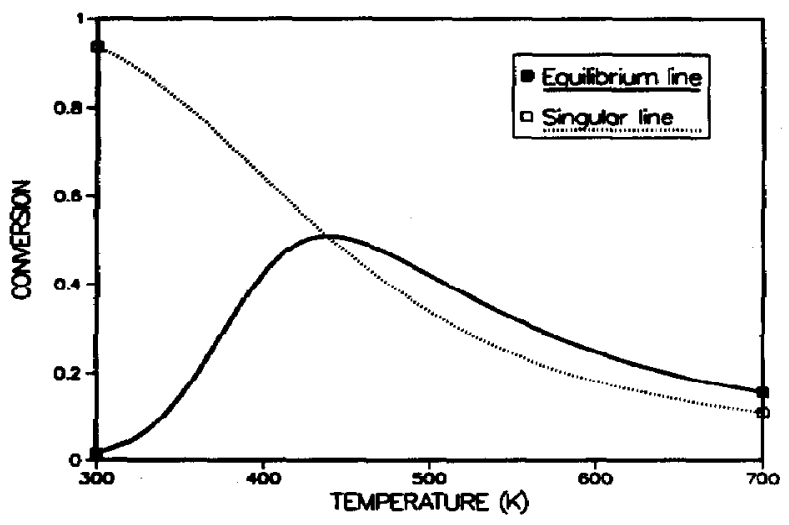

Fig. 10. Equilibrium diagram and singular line for the CSTR.

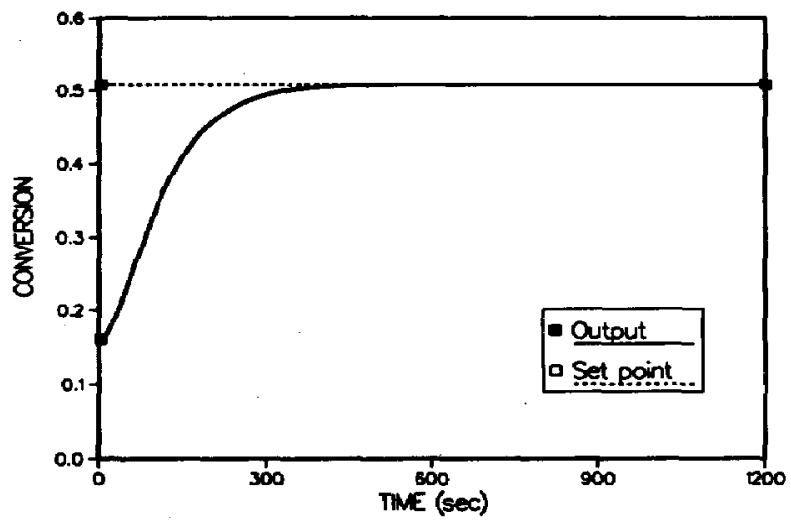

Fig. 11. Conversion profile for set-point change (a).

side of the conversion maximum in Fig. 10. The control objective is to bring the reactor to the desired conversion. Figure 11 illustrates the output profile under the controller of eq. (16), while Fig. 12 illustrates the trajectory in the conversion/temperature diagram. As established by Economou and Morari (1985), even a linear controller can perform satisfactorily in this case. In the second run, the process is assumed to be at the steady state $x_{1 \mathrm{~s}}=0.59 \mathrm{gmoll}^{-1}$, $x_{2 s}=0.41 \mathrm{gmol1}^{-1}$ and $x_{3 s}=504 \mathrm{~K}$, which corresponds to a point at the right side of the conversion maximum in Fig. 10. The control objective is again to bring the reactor back to the desired conversion. As shown by Economou and Morari (1985), because of a sign change in the steady-state gain in this operating

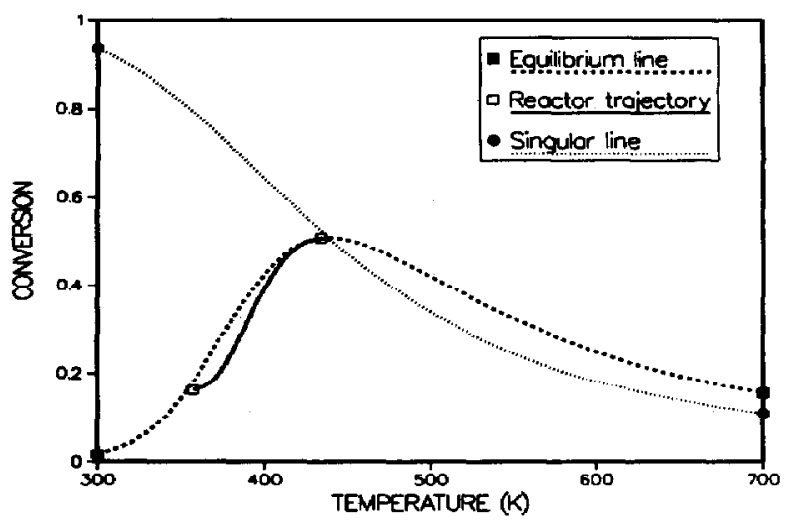

Fig. 12. Reactor trajectory in the conversion/temperature diagram for set-point change (a).

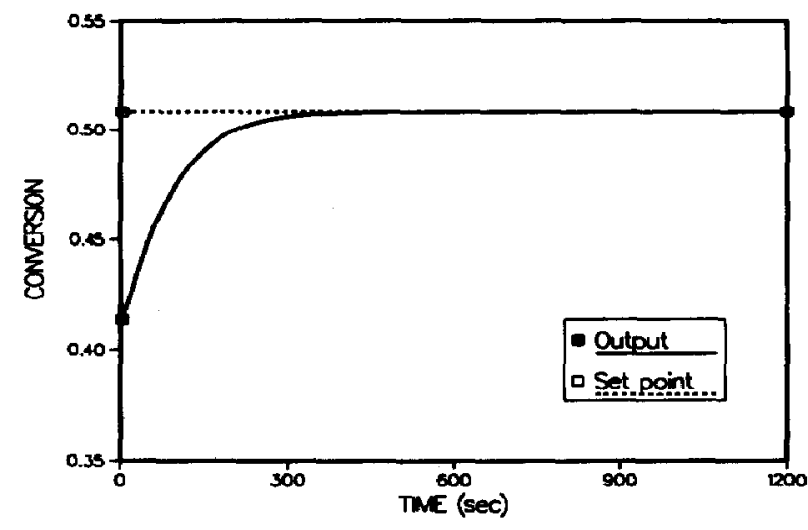

Fig. 13. Conversion profile for set-point change (b).

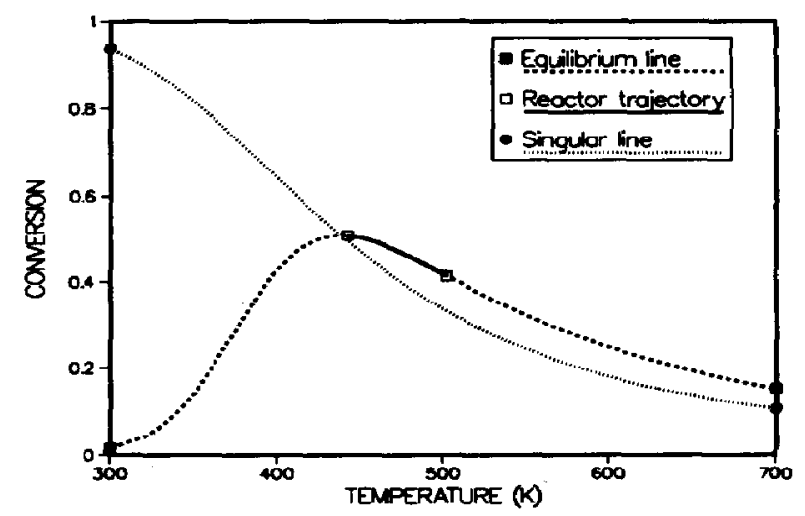

Fig. 14. Reactor trajectory in the conversion/temperature diagram for set-point change (b). 
region, any linear controller with integral action leads to instability, while a proportional linear controller leads to offset. On the other hand, as shown in Fig. 13, the nonlinear controller of eq. (16) induces the theoretically predicted output response, controlling the process to its set point. Figure 14 illustrates the corresponding trajectory in the conversion/temperature diagram. As expected, the results presented here using the derived analytical control laws are in complete agreement with the results obtained by Economou and Morari (1985), where the numerical IMC algorithm was employed for the same cases.

Acknowledgement-Financial support from the National Science Foundation, Grant No. CTS-8912836, is gratefully acknowledged.

\section{NOTATION}

$c_{A O}$

$c_{A}, c_{B}$

$c_{p}$

C

$E_{A}, E_{B}$

$f$

$g$

$\boldsymbol{h}$

$-\Delta \boldsymbol{H}$

$k_{A}, k_{B}$

$\boldsymbol{L}_{f} \boldsymbol{h}$

$L_{f}^{k} h$

P

$Q$

$r$

R

$\mathbb{R}$

$\mathbb{R}^{n}$

s

$t$

$T$

$T_{0}$

$\boldsymbol{u}$ $v$

$w$

$x$

$x_{m}$

$y$

$y_{m}$

$y_{s p}$

$y^{*}$

$z$ auxiliary variable

state vector of full-order process inverse state vector of process

state vector of process model

process output

process model output

output set point

auxiliary variable

state vector of minimal-order process inverse

Greek letters

$\boldsymbol{\beta}_{\boldsymbol{k}} \quad$ adjustable parameters

$\gamma_{k} \quad$ adjustable parameters

$\zeta$

5

$\rho$

$\tau$ state variables in the controllers density of the reacting mixture, $\mathrm{kg}^{-1}$ reactor residence time, $s$

\section{REFTRENCES}

Bartee, J. F., Bloss, K. F. and Georgakis, C., 1989, Design of nonlinear reference system control structures. AIChE Annual Meeting, San Francisco, CA.

Byrnes, C. I. and Isidori, A., 1985, Global feedback stabilization of nonlinear systems, in Proceedings of the 24th IEEE CDC, Ft. Lauderdale, FL, p. 1031.

Cheng, C. M. and Brosilow, C. B., 1987, Model predictive control of unstable processes. AIChE Annual Meeting, New York.

Desoer, C. A. and Vidyasagar, M., 1975, Feedback Systems: Input-Output Properties. Academic Press, New York.

Economou, C. G. and Morari, M., 1985, Newton control laws for nonlinear controller design, in Proceedings of the 24th IEEE CDC, Ft. Lauderdale, FL, p. 1361.

Economou, C. G., Morari, M. and Palsson, B. O., 1986, Internal model control 5 . Extension to nonlinear systems. Ind. Engng Chem. Proc. Des. Dev. 25, 403.

Henson, M. A. and Seborg, D. E., 1991, An internal model control strategy for nonlinear systems. A.I.Ch.E. J. 37, 1065.

Hidalgo, P. M. and Brosilow, C. B., 1990, Nonlinear model predictive control of styrene polymerization at unstable operating points. Comput. chem. Engng 14, 481.

Hirschorn, R. M., 1979, Invertibility of nonlinear control systems. SIAM J. Control Optimization 17, 289.

Kravaris, C. and Chung, C. B., 1987, Nonlinear state feedback synthesis by global input/output linearization. A.I.Ch.E. J. 33, 592.

Parrish, J. R. and Brosilow, C. B., 1988, Nonlinear inferential control. A.I.Ch.E. J. 34, 633.

Safonov, M. G., 1980, Stability and robustness of multivariable feedback systems. MIT Press, Cambridge, MA. 